\title{
Kasabach Merrit Syndrome in a Third Trimester Pregnancy
}

\author{
SAMARATH SHUKLA, ${ }^{1}$ NEEMA ACHARYA, ${ }^{2}$ SOURYA ACHARYA ${ }^{3}$ DP RAI PUT, ${ }^{4}$ S VAGHA ${ }^{5}$
}

\begin{abstract}
:
The case report deals with a third trimester pregnant female who presented with a mass in the dorsal paraspinal region (D7) resulting in rapid onset paraparesis gradually increasing in severity. The mass was of vascular nature (Haemangioma) on MRI, which on histopathology was confirmed as infiltrating ingiolipoma. Complications in haemangiomas occur and one such being the "Kasabach Merritt syndrome" consisting a triad of thrombocytopenia, haemangioma and coagulopathy, also dubbed as the "Haemangioma Thrombocytopenia Syndrome". But its presentation in third trimester is definitely an unusual presentation considering the fact that such thrombocytopenic syndromes usually occur with congenital haemangiomas and with other benign vascular lesions in infants. The present case deals with the mass in dorsal spinal area resulting in compressive myelopathy as well as thrombocytopenic coagulopathy.
\end{abstract}

Keyword: Paraspinal, Paraparesis, Haemangioma, MRI, Angiolipoma, Thrombocytopenia, Coagulopathy, Myelopathy, Kasabach Merritt Syndrome

\section{Introduction:}

Conventional lipomas are benign capsulated tumors, Histological variants as in with lipomas included are areas of bone formation (osteolipoma), or areas with vascular elements admixed with matures adipose tissue (angiolipoma), cartilage (chondrolipoma), abundant fibrous tissue (fibrolipoma), or extensive myxoid change (myxolipoma). Angiolipomas are relatively common and usually appear in the late teens or early twenties. Children and patients older than 50 years are rarely involved. There is a male predominance and an increased familial incidence has been described ( $5 \%$ of all cases). ${ }^{1,2}$ Mode of inheritance is not clear.

A variety of lipomatous tumor variants may show infiltrative behavior with lack of capsule formation. Intramuscular lipoma, shows an infiltrative growth pattern with mature adipocytes infiltrating and encasing skeletal muscle fibres that often show evidence of atrophy. Angiolipoma especially the ones present in paraspinal and spinal location are more aggressive in their behavior and found to infiltrate into the surrounding tissue rather than their more indolent benign capsulated forms. In lipoma the subsynovial connective tissue is infiltrated by mature adipocytes; scattered inflammatory cells are also usually present. ${ }^{3}$

Angiolipomas are always benign and show no tendency to recur. Malignant transformation does not occur. The forearm is the most common site, followed by the trunk and upper arm. Spinal angiolipomas and intramuscular haemangiomas, previously also called 'infiltrating angiolipomas', are different lesions ${ }^{3,4}$ Considering the genetic make up the angiolipomas are more intricately linked with haemangiomas rather than lipomas. And hence all cases of angiolipomas are to have tilt towards the clinical as well as gross behavioural aspects of that of a haemangioma. Complications of haemangiomas can be ones commonly come across including super added infection, haemorrahge to rare situations of compression, as well as thrombocytopenia. The combination of hemangioma, thrombocytopenia, and coagulopathy is termed KasabachMerritt Syndrome (KMS). ${ }^{5}$

The present case scenario deals with a female patient in her third trimester pregnancy with bilateral rapid onset paraparesis with gradual increasing severity and ecchymotic patches in both lower limbs. It was only when all infective causes (especially viral haemorraghic fever) were ruled out through elaborate investigative workup, that a MRI spine (thoracic) was advised which revealed a paraspinal dorsal mass, an entity known as "Infiltrating angiolipomas" on histopathology was confirmed, leading to a case scenario of Kasabach Merritt Syndrome.

1. Associate. Professor, Dept. of Pathology, JNMC, Wardha

2. Associate. Professor, Dept. of Obst \& Gynec. JNMC, Wardha

3. Associate Professor, Dept. of Medicine, JNMC, Wardha

4. Assistant Professor, Dept. of Medicine, LNMC, Bhopal

5. Professor \& Head, Dept. of Pathology, JNMC, Wardha

Correspondence: Dr. Samarth Shukla Assoc. Prof, Dept. of Pathology, J N Medical College, DMIMS Univ, Sawangi (Meghe), Wardha, Email:samarthshukla@hotmail.com 


\section{Case History:}

A 20 year old primigravida with full term pregnancy (40weeks) presented to medicine OPD with complaints of sudden onset of weakness in both lower limbs since 4 days and urinary incontinence for1 day. There was no history of fever, trauma, diarrhoea, sensory loss,seizures or unconsciousness. There was no past history of similar complaints. On examination her vital signs were stable and pallor, icterus, cyanosis, lymphadenopathy were absent. Ecchymotic patches were present in both lower limbs. (Fig-1) CVS and RS examination was unremarkable. Per abdomen examination revealed a full term single live intrauterine fetus with cephalic presentation which was non engaged due to cephalopelvic disproportion.

In CNS examination, higher psychic functions were normal. There were no cranial nerve abnormalities. Both upper limbs were normal. Motor system examination of lower limbs revealed hypotonia. Power was grade $3 / 5$ in hip flexors and extensors, grade 2/5 in ankle dorsiflexors. Coordination couldn't be tested. Both knee and ankle reflexes were absent and both planters were extensors. Abdominal reflexs were absent in lower quadrants. Beevor's sign was absent. Sensory system examination revealed diminution of pain and temperature senses below umbilicus (on asking leading question she told that sensory loss is approximately reduced $40 \%$ of normal). There was no spinal tenderness, paravertebral mass or gibbus. She was catheterized. A provisional diagnosis of acute onset, gradually progressive, sensorymotor UMN type of paraparesis with neuronal shock, with bladder involvement, level being D 10 segmental and cause being transeverse myelitis was kept. Empirically IV dexamethasone $8 \mathrm{mg} 8$ hourly was started.

Investigations revealed $\mathrm{Hb}-10$ gm\%, TLC- 5500/mm3, DLCpolymorphs $66 \%$, lymphocytes $32 \%$, monocytes $2 \%$.

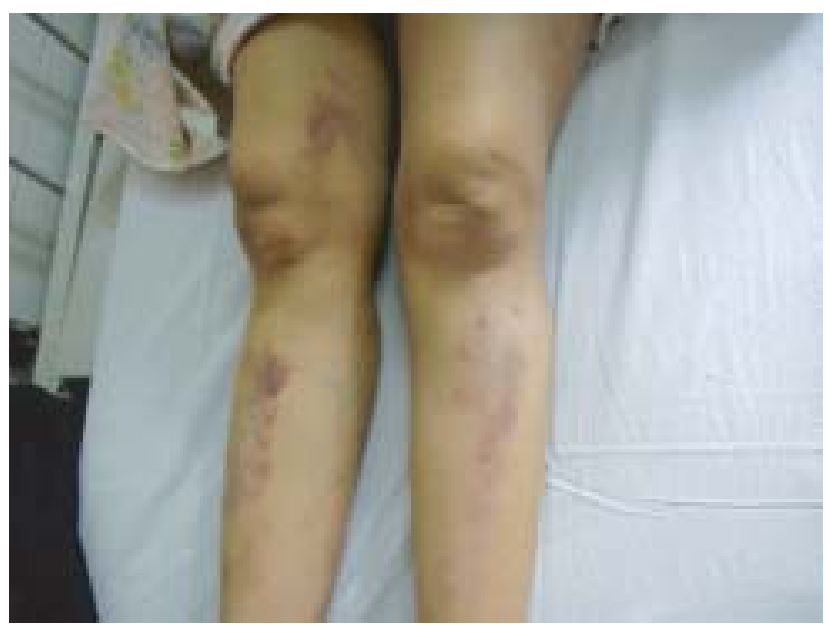

Fig-1: Echymotic patches in lower limbs

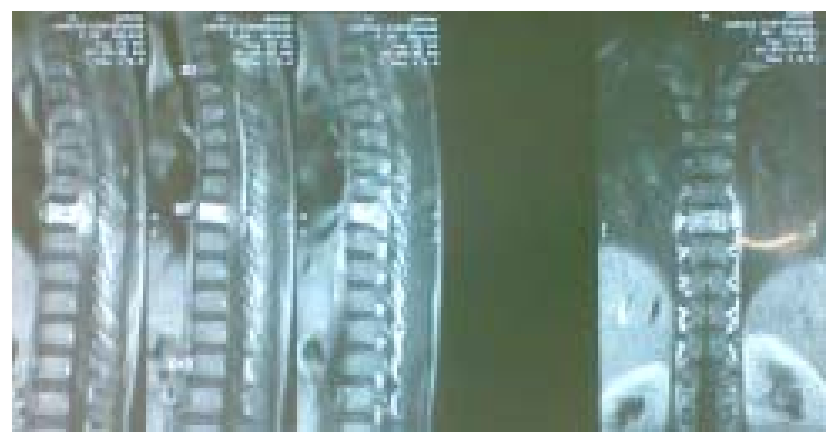

Fig-2: MRI of spine showing diffuse signal density changes noted at D7 vertebra.

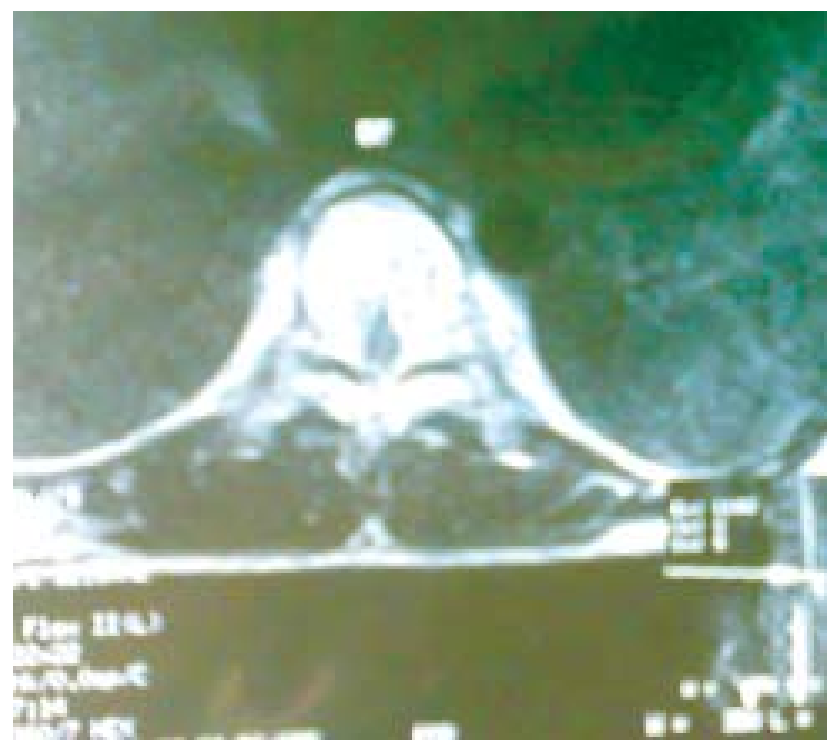

Fig-3: MRI of spine showing intact vertebral body

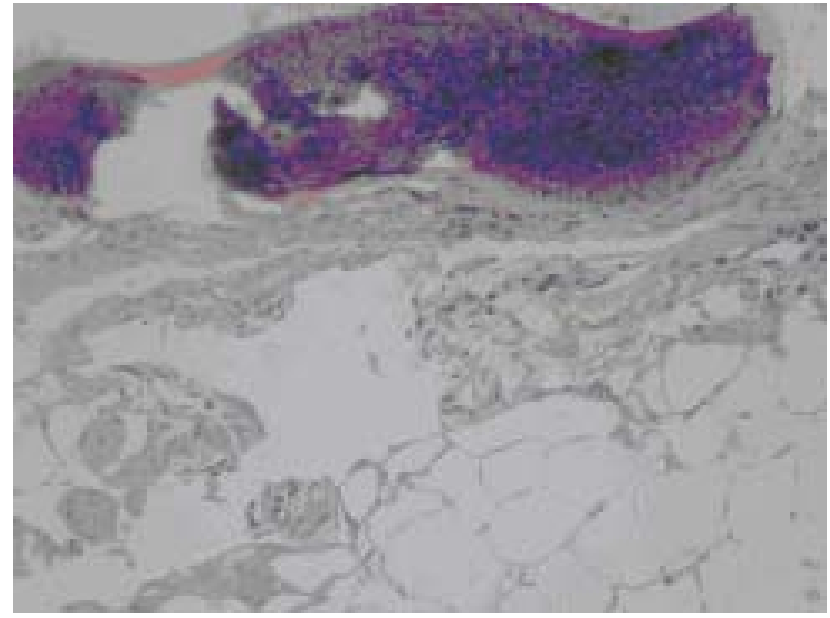

Fig.-4: Histopathology of sectioned tissue showing hyaline thrombi in lumen without definite capsular pattern

Absolute platelet count was 48,000/mm3, APTT, PT, Thrombin time were normal. ESR- $12 \mathrm{~mm}$ in 1st hour. Urine examination was normal. Serum ANA and pANCA was negative. Obstertric sonography was normal. MRI of spine 
revealed diffuse signal density changes noted at D7 vertebra with maintained vertebral morphology, prominence of secondary trabeculae within vertebra. (Fig-2, Fig-3) The lesion showed involvement of pedicle with expansion of pedicle causing compression of cord and mild cord oedema noted. There was no bony obstruction or paradiscal involvement.

With consultation with neurosurgeon and orthopaedic surgeon decision of delivery of the fetus followed by decompression of cord in a separate setting was made. She was transfused with 6 units of platelets after which her platelet count was $10,2000 / \mathrm{mm}^{3}$. Ten days after caeserian section laminectomy at D7 level was done and paravertebral mass of $3 / 3 \mathrm{~cm}$ was excised and sent for histopathological examination.

The specimen (tumor mass) sent for histopathology was grossed in the surgicopathological lab, the slides stained with $H \& E$ stain revealed sections showing admixture of mature adipose tissue amidst benign vascular proliferation (hamartomatous), at places non specific chronic inflammatory infiltrate can be appreciated. One or two blood vessels show hyaline thrombi in their lumen , this being a pathognomic feature on histopathology of angiolipoma, the histopathology section characteristically lacked peripheral fibrous tissue layer(capsule) suggesting and retariating the uncapsulated infiltrating nature of the mass.(Fig-4)

Physiotherapy was started. Patients power improved to 4/6 after 1 month, bladder symptoms recovered to normal within 15 days of surgery. Ecchymotic patches resolved, Absolute platelet count was $250,000 / \mathrm{mm}^{3}$. She is awaiting second follow up after 1 month.

\section{Discussion:}

Spinal angiolipomas are distinct, benign lesions composed of mature lipocytes admixed with abnormal blood vessels. ${ }^{6}$ They account for $1.2 \%$ of all spinal axis tumours. They can be regarded as maldevelopment, neoplastic or hamartomatous diseases. The hypothesis of hamartomatous origin from primitive pluripotential mesenchymal cells is gaining wide acceptance. Spinal epidural angiolipomas predominantly affect women. Their preponderance in older, peri-, or postmenopausal women and their clinical exacerbation in pregnant women support a role of hormonal influence. ${ }^{6}$ About $22 \%$ of females are initially symptomatic during the last trimester of pregnancy, and coincides with the history of weight gain. ${ }^{6}$ At the time of diagnosis, most patients show paraparesis with hyperreflexia, bilateral extensor plantars, with loss of pain, temperature, vibration, and position sensation.
Infiltrating angiolipomas are rare, benign neoplasms found most frequently in the soft tissues of the thigh. The clinicopathologic characterization of the infiltrating lngiolipomas was carried out by Gonzalez-Crussi, Enneking, and Arean. ${ }^{7}$ Histologically, these unencapsulated tumors are composed predominantly of mature lipocytes with foci of angiomatous proliferation. Mitoses and cellular atypia are absent and the lesion does not metastasize. ${ }^{7}$ In contrast to the noninfiltrating angiolipomas, which are well defined, encapsulted, and subcutaneous, infiltrating angiolipomas are locally aggressive. They infiltrate through muscle, fascia, and subcutaneous tissues with apparent disregard for anatomic barriers, and can progress to neurologic involvement or bone destruction. ${ }^{8}$

The fact that angiolipomas lack chromosomal aberrations(like haemangiomas and unlike lipomas) suggest that they are haemangiomas with fat rather than true mixed tumors. ${ }^{8}$ The so called infiltrating angiolipomas are probably not true mixed tumors but rather intramuscular large vessel haemangiomas in which the proportion of the affected muscle tissue have been replaced by fat. Distension of the vascular channels due to a $30-50 \%$ increase in circulating blood volume increased venous distensibility due to maternal production of progesterone, and the endothelial growth promoting effect of estrogen are the other factors implicated. The overall complication rate of hemangiomas ranges from $4.51 \%$ to $19.7 \% .{ }^{9}$ A very rare manifestation in terms of complications includes the Kasabach Merritt syndrome. Kasabach-Merritt syndrome (KMS), also known as "Hemangioma with thrombocytopenia”) is a rare disease, usually of infants, in which a vascular tumor leads to decreased platelet counts and sometimes other bleeding problems, which can be lifethreatening. ${ }^{3,10}$ It is also known as hemangioma thrombocytopenia syndrome. It carries the names of Dr Haig Haigouni Kasabach and Dr Katharine Krom Merritt, the two pediatricians who first described the condition in 1940.11

KMS is usually caused by a hemangioendothelioma or other vascular tumor, often present at birth. Although these tumors are relatively common, it is rare for them to cause KMS. When these tumors are large or are growing rapidly, sometimes they can trap platelets, causing severe thrombocytopenia. The combination of vascular tumor and consumptive thrombocytopenia defines KMS. Tumors can be found in the trunk, upper and lower extremities, retroperitoneum, and in the cervical and facial areas. ${ }^{10}$

The present case deals with two extremely rare yet very significant complications with respect to the behavior of the angiolipoma (gross as well as the microscopic). The paraspinal mass impinged on the spinal cord thereby not 
only producing local pressure symptoms but also causing obvious and progressive myelopathy. Microscopically, it also caused platelets consumption and resulting coagulative thrombocytopenia. The entire case scenario interestingly deals with outcomes of pregnancy related vascular proliferations and their behavioural patterns.

Successful surgical management of infiltrating angiolipoma depends on the anatomic location and extent of the lesion. The local aggression of the lesion requires an excision with wide margins, but its benign natural history generally precludes amputation or excessive loss of function.

\section{Conflict of Interest: None}

\section{References:}

1. Bordet R, Ghawche F, Destee A. [Epidural angiolipoma and multiple familial lipomatosis]. Rev Neurol (Paris) 1991; 147: $740-742$

2. Cina SJ, Radentz SS, Smialek JE. A case of familial angiolipomatosis with Lisch nodules. Arch Pathol Lab Med 1999; 123: 946-948

3. Hamakawa H, Hino H, Sumida T, Tanioka H. Infiltrating angiolipoma of the cheek: a case report and a review of the literature. J Oral Maxillofac Surg 2000; 58: 674-677
4. Turgut M. Spinal angiolipomas: report of a case and review of the cases published since the discovery of the tumour in 1890. Br J Neurosurg1999;13: 30-40

5. James W, Berger T, Elston D. Andrews' Diseases of the Skin: Clinical Dermatology. (10th ed.). 2005, Saunders.

6. Preul MC, Richard Leblanc, Donatella T, Yves R, Ronald P. Spinal anpolipomas. Report of three cases. J Neurosurg, 1993; 78:280-286

7. Gonzalez-Crussi F, Enneking WF, Arean VM. Infiltrating angiolipoma. J Bone Joint Surg [Am] 1966; 48 : 1111 -1124

8. Sciot R et al. cytogenetic analysis subcutaneous angiolipoma : further evidence supporting it's difference from ordinary pure lipomas: a report of the CHAMP study group. Am J Surg Pathol 1997; 21:441-444

9. Creasy C, Flickinger F, Kraus R. Maternal liver haemangioma in pregnancy as an incidental finding. Obstet Gynaecol 1985; 66 : 105-148

10. Hall G. Kasabach-Merritt syndrome: pathogenesis and management. Br J Haematol 2001;12 (4): 851-62

11. Kasabach HH, Merritt KK. Capillary hemangioma with extensive purpura: report of a case. Am J Dis Child 1940; 59: 1063 